\title{
TERAPIA OCUPACIONAL EN NEONATOLOGÍA: UNA EXPERIENCIA DESDE EL SUR
}

\author{
OCCUPATIONAL THERAPY IN NEONATAL CARE UNIT: AN \\ EXPERIENCE FROM THE SOUTH
}

\section{Clara Avilès ${ }^{1}$}

\section{Resumen}

A pesar del objetivo central de terapia ocupacional de fomentar un óptimo desempeño ocupacional de nuestros usuarios en todas las etapas vitales, nuestra presencia en la atención de bebés prematuros en Unidades de Neonatología ha sido parcial hasta ahora, a pesar de que contamos con herramientas terapéuticas que nos facultan para entregar un aporte valorable en dicha población.

El presente artículo describe la experiencia de la sección de terapia ocupacional niños del Servicio de Medicina Física y Rehabilitación en la atención de bebés prematuros hospitalizados en la Unidad de Neonatología del Hospital Guillermo Grant Benavente de Concepción.

Es necesario que los terapeutas ocupacionales nos planteemos una constante puesta en común, análisis y reflexión acerca de nuestro trabajo en esta área en particular, tanto con el propósito de contribuir al logro de los mejores niveles de desarrollo integral de estos niños, como de potenciar nuestro progreso y posicionamiento profesional.

\section{Palabras claves}

Neonatología, Prematuridad, Terapia Ocupacional, Desarrollo Psicomotor.

\begin{abstract}
In spite of occupational therapy central target related to promote an ideal occupational performance in our patients from all ages, our presence in neonatology in preterm attendance had been partial until the present, even when the therapist have terapeutics tools that authorize us to give an import help to that population. The present paper describe the experience from the pediatric occupational therapy, as part of the Physical Medicine and Rehabilitation Service in the attention of preterm babies hospitalized in the Neonatology Unit at the Hospital Guillermo Grant Benavente in Concepcion.
\end{abstract}

1 Terapeuta Ocupacional, Unidad de Neonatología Hospital Guillermo Grant Benavente de Concepción. Chile. 
It is necessary that the occupational therapist explained us an constant updating, analisis and reflexion about our work in this particular area, with the propose to contribuit to obtain the much higher levels in the integral development from the children, as well as our progress and to promote our professional position.

\section{Keywords}

neonatology, prematurity, occupational therapy, psicomotor development.

\section{INTRODUCCIÓN}

Si bien en los países desarrollados la intervención de terapia ocupacional en unidades de neonatología data de aproximadamente 20 - 30 años, en nuestro medio ésta constituye un área insuficientemente cubierta, a pesar del rol que corresponde a los terapeutas ejercer en beneficio de los niños allí atendidos. Así, a pesar de la existencia de algunas experiencias, aún no hemos logrado conformar una labor sistematizada y reconocida por los equipos profesionales tradicionales (pediatras, matronas, enfermeras). Quizás esto se deba, en parte, a que los terapeutas con frecuencia tenemos dificultad para explicar con precisión en qué consiste nuestro quehacer profesional; también podemos entenderlo por el reconocimiento de que aquellas son unidades en las que, debido a la naturaleza de su accionar, resulta indispensable para los profesionales adquirir un conocimiento especializado en base al cual sustentar su colaboración.

Por estos motivos resulta útil difundir las experiencias hasta ahora elaboradas con el fin de promover un diálogo que se traduzca en una ampliación de nuestro quehacer en esta primera y crucial etapa de la vida.

Esto es particularmente válido en relación a la atención de bebés nacidos de pretérmino, los que debido a su particular condición se encuentran en riesgo de evolucionar con problemas motores, sensoriales y del lenguaje, entre otros, así como de sufrir los efectos adversos del medio ambiente altamente tecnologizado de las unidades de neonatología, con las consecuencias que aquellos pueden tener en su desarrollo psicomotor y capacidad funcional a mediano y largo plazo.

En efecto, la literatura especializada ha mostrado de manera contundente de qué manera el hecho de encontrarse fuera de su ambiente natural -el vientre materno- en un período crítico de su desarrollo fisiológico y neurológico, y verse expuesto a un ambiente adverso pleno de estímulos perjudiciales para el bebé, se traduce en alteración de su desarrollo neurológico y psicomotor, situación que resulta más perjudicial cuanto menor sea la edad gestacional en que nace el niño.

Los terapeutas ocupacionales tenemos una labor específica que cumplir en la atención de estos niños, la que puede resumirse en la aplicación de intervenciones destinadas a adecuar los estímulos del ambiente junto con apoyar los esfuerzo de autorregulación del niño, con el fin de favorecer un adecuado desarrollo de funciones y estructuras corporales, de modo de contribuir a que el bebé pueda adquirir las habilidades necesarias para asegurar un desempeño ocupacional exitoso en el mediano y largo plazo.

El objetivo de este estudio consiste en dar a conocer una experiencia de trabajo de terapia ocupacional en la Unidad de Neonatología del Hospital Guillermo Grant Benavente de Concepción, la que se ha ejecutado en forma regular durante los últimos 6 años.

\section{ANTECEDENTES}

\section{Desarrollo neurológico y neuromotor del bebé prematuro}

En la vida intrauterina el bebé en desarrollo permanece en un ambiente cálido, quieto, libre de estímulos visuales y auditivos (o estos últimos son muy atenuados); en posición de flexión global creciente, con un 
espacio que provee contención, limita y controla los movimientos de las extremidades, siendo posible para el bebé efectuar movimientos de extensión pero volviendo siempre a la flexión y a la línea media. El feto evidencia movimiento a partir de la semana 7 - 9 de gestación, movimiento espontáneo influido por programas motores que favorecen incluso eventualmente la posibilidad de llevar sus manos a la boca para succionar sus dedos. El niño recibe principalmente estimulación táctil proveniente del contacto con las paredes del útero (el que además controla el efecto de la fuerza de gravedad), estimulación vestibular determinada tanto por los cambios de posición que él mismo efectúa como por los movimientos corporales de la madre, y estimulación propioceptiva proveniente de sus propios movimientos y posiciones corporales. Duerme la mayor parte del tiempo y su organismo en desarrollo paulatinamente adquiere la capacidad para incorporar, en forma secuencial a partir del nacimiento, estímulos controlados y adecuados.

Esas condiciones, a la par del natural equilibrio fisiológico, conforman la base natural para un correcto desarrollo psicomotor del niño. La posición de flexión global intrauterina favorece el tono flexor propio de los bebés de término sanos, que los predispone en forma óptima para el futuro desarrollo de habilidades motoras gruesas y finas. El tipo de estimulación táctil descrito es el adecuado para las características de su piel, así como la ausencia de estímulos visuales favorece el equilibrio homeostático en el bebé. A partir de su nacimiento se verá incorporado a un ambiente acogedor -la familiaque lo nutrirá y proveerá todas las condiciones para asegurar un desarrollo normal en todas las esferas.

\section{Efectos del nacimiento prematuro}

El bebé nacido de pre término se encuentra en una condición de vulnerabilidad al continuar su gestación fuera del vientre materno, con un SNC que no se encuentra en condiciones de enfrentar y organizar los estímulos provenientes de un ambiente más bien desfavorable. Junto con todo lo que el nacimiento prematuro implica en términos de su condición vital, queda expuesto en forma repentina a una serie de estímulos que ejercen efectos dañinos en el corto, mediano y largo plazo. Pierde la protección que le brindaba el útero siendo expuesto a la fuerza de gravedad y a la pérdida de la natural posición de flexión global. Debido a ello, así como a la ausencia de elementos de contención, el bebé efectúa movimientos que lo llevan más bien a posiciones de extensión y de aplanamiento sobre las superficies de cunas e incubadoras. Así, por la ausencia de condiciones de flexión, contención y orientación a la línea media, se genera un desequilibrio de la acción muscular del bebé.

Los procedimientos médicos y de enfermería pueden requerir frecuentes manipulaciones que implican estimulación táctil adversa, con eventual generación de dolor. Además el niño permanece en un ambiente con luz y ruido constantes, lo que unido a lo anterior se traduce en la pérdida o alteración del establecimiento de ritmos de sueño-vigilia.

La situación descrita implica varios efectos negativos para el desarrollo neurológico y psicomotor del niño, con consecuencias adversas en el corto, mediano y largo plazo, las que se verán intensificadas cuanto menor sea la edad gestacional en que se produce el nacimiento. Existen numerosos estudios que corroboran esta afirmación. Hubner (1) encontró que en niños nacidos con menos de 26 semanas de gestación es posible detectar hasta un $25 \%$ de déficits mayores, tales como parálisis cerebral y problemas cognitivos. En su artículo plantea que a los 8 años de edad, un alto porcentaje de niños que al nacer pesaron menos de 1000 grs. requerían apoyo educativo. Un factor crítico a juicio de esta autora sería la presencia de hemorragia intraventricular, cuya incidencia es mayor a medida que disminuye la edad gestacional con la que nace el niño. Habría también un porcentaje importante de déficits menores, tales como alteraciones del tono muscular, de la postura, de los reflejos y alteraciones visuales leves.

Durante los primeros meses de edad corregida en los niños prematuros es posible observar la presencia de signos neurológicos adversos en las áreas motora 
gruesa (como retracción escapular, dificultad para llevar manos a línea media, tendencia al aumento de tono extensor, dificultad para tolerar la posición prona) y fina (tendencia a incluir pulgar) junto con preferencia por girar la cabeza mayormente hacia el lado derecho; así como manifestaciones sugerentes de alteración en su capacidad de modular estímulos del medio.

Numerosos estudios plantean que los prematuros nacidos con menos de 1500 grs. de peso tendrían diversos grados de alteraciones motoras, cognitivas, sensoriales, del lenguaje, así como déficit atencional y problemas de aprendizaje.

De acuerdo a informe del Ministerio de Salud, entre los niños nacidos de menos de 1.500 grs. habría un $23 \%$ de secuelas neurológicas (2); un $42 \%$ de los niños con hemorragia intracraneana grados III y IV evolucionan con parálisis cerebral, pudiendo haber otros problemas motores de menor gravedad pero que igualmente afectan la funcionalidad y el desarrollo psicomotor del niño. Aproximadamente un $8 \%$ presentaría problemas de lenguaje.

Otros estudios establecen la presencia de alteraciones motoras y neurológicas de diverso grado. Connors (3) plantea que a los 4 meses de edad corregida en los niños con antecedente de prematuridad es posible observar asimetría de cabeza y tronco, preferencia por girar la cabeza a la derecha, movimientos arrítmicos de extremidades, sobresalto ante cambios de posición, discreta resistencia a la movilización pasiva, e insuficiente control de cabeza. En tanto que a los 8 meses de edad corregida los niños pueden presentar leve hipertonía o hipotonía, dificultad para pasar de sedancia a gateo o viceversa, falla en movimientos recíprocos durante el gateo, ocasionales patrones de movimiento anormal aun en presencia de tono muscular normal. Estos problemas suelen ser transitorios y de buen pronóstico en presencia de adecuado manejo de rehabilitación. Sin embargo, de acuerdo a la autora, sería posible detectar su presencia en algunos niños a los 18 meses de edad corregida, además de patrones de prehensión inmaduros, asimetría en el uso de ambas manos y disminución de las reacciones de equilibrio.
Estos déficits pueden expresarse también como retraso para adquirir el gateo y la marcha, preferencia marcada por usar una mano (generalmente la derecha, lo que podría relacionarse con su tendencia desde el nacimiento por girar la cabeza a la derecha), así como limitada capacidad para mantener su atención en actividades de percepción-manipulación.

La literatura describe ampliamente las dificultades que pueden presentar niños con antecedente de prematuridad en el ámbito escolar, debido a eventuales problemas de coordinación, de aprendizaje y/o por problemas de déficit atencional. Foulder-Hugues encontró, en un estudio en que comparó a niños de pre término con sus pares nacidos de término en los primeros años de escolaridad, que los primeros evidenciaban peores resultados en evaluaciones motoras (motricidad gruesa y fina) y de integración visomotora, con todo lo que ello implicaba en términos de déficits de control postural y lectoescritura, entre otras manifestaciones. Así mismo los niños con antecedentes de prematuridad tenían un desempeño académico más deficiente, a pesar de presentar $\mathrm{Cl}$ normal (4).

Un estudio efectuado sobre un grupo de niños nacidos con un promedio de 30 semanas de gestación, mostró que el repertorio de movimientos que estos presentaban a edades tempranas se relacionaba con su situación en la edad escolar (5).

Otras investigaciones destacan la presencia de alteraciones conductuales como condicionantes de bajo rendimiento en la edad escolar, junto con eventuales dificultades en tareas visuoespaciales y de cálculo, así como problemas de lenguaje (6).

El nacimiento prematuro puede suponer un efecto negativo para el normal establecimiento del vínculo padres-hijo, con todo lo que esto implica para el desarrollo del niño. El hecho de verse abruptamente separados de su hijo recién nacido, unido a las condiciones vitales de éste y a las restricciones que el funcionamiento de las unidades de neonatología implican para la presencia de los padres, condiciona una situación de deprivación para el niño junto con una sensación de incompetencia por parte de los padres, que pueden tener dificultades 
para comprender el manejo médico y el lenguaje técnico inherente al mismo. La mayoría de los padres tienden a sentir, en las primeras semanas posteriores al nacimiento, que carecen de las habilidades necesarias para atender adecuadamente a su hijo y cumplir su rol de padres (7), situación que puede constituir también un factor de riesgo para el normal desarrollo del niño a futuro si no es resuelta en forma oportuna.

\section{INTERVENCIÓN DE TERAPIA OCUPACIONAL}

\section{Fundamentación teórica}

La intervención de terapia ocupacional en Unidades de Neonatología se justifica a partir de la comprensión de la necesidad de proteger al bebé y favorecer su desarrollo psicomotor, en cuanto esta primera etapa de su vida conforma la base sobre la cual se estructurará su desarrollo global a futuro y el consecuente desempeño funcional del niño.

Esta intervención debe sustentarse en un enfoque protectivo y preventivo, orientado en primera instancia (8) a proteger al niño de estímulos excesivos o inadecuados, facilitar o proveer estímulos adecuados para facilitar su capacidad de organizar la información sensorial y producir una respuesta adaptativa con el fin de favorecer su estabilidad fisiológica en el presente y adecuado desarrollo psicomotor y neurológico en el futuro. Junto con favorecer la génesis de respuestas adaptativas, para la terapia ocupacional un punto focal se refiere a facilitar la participación del bebé en ocupaciones adecuadas para su edad, la que en esta etapa y una vez sobrepasado el período crítico, consiste en comunicar sus necesidades (9). Para ello una condición indispensable para el profesional consiste en observar e interpretar la conducta del bebé.
La modalidad de intervención denominada $\mathrm{NI}$ DCAP, Newborn Individualizad Developmental Care and Assessment Program, ${ }^{2}$ diseñada a partir de la Teoría Sinactiva del Desarrollo formulada por Heidelise Als, y cuyo fin consiste en facilitar la adaptación extrauterina del neonato y proteger su estabilidad fisiológica, conforma en la actualidad uno de los enfoques más ampliamente utilizados para estructurar intervenciones en Unidades de Neonatología, por cuanto su aplicación ha demostrado amplios beneficios para el logro de un desarrollo psicomotor normal (10).

Otro enfoque teórico que constituye un adecuado fundamento para elaborar programas y modalidades de intervención en esta área es la teoría de Gorski y Brazelton que describe 3 estados o etapas de los bebés prematuros: In turning (hacia adentro), coming out (saliendo) y reciprocity (reciprocidad activa). En la primera etapa el niño tiene muy poca o ninguna capacidad de interactuar con el medio y centra su esfuerzo en lograr estabilidad fisiológica. En la segunda etapa, afianzada ya en gran medida su estabilidad, puede incorporar algunos estímulos siempre que éstos se presenten en modalidad, cantidad e intensidad adecuados; en la tercera etapa tiene mayor capacidad para autoregularse y esbozar algunas respuestas funcionales (11).

\section{Experiencia en el Unidad de Neonatología}

El Hospital Guillermo Grant Benavente de Concepción es un hospital tipo 1 que constituye un centro de referencia de la zona sur del país para la atención de diversas patologías. Su Unidad de Neonatología forma parte de la Red Neonatal Chilena dependiente del Ministerio de Salud y a ella se derivan niños provenientes desde la Séptima hasta la Décima Región.

Nuestra experiencia en esta unidad se inició a fines de 2004 - comienzos de 2005, y en sus orígenes se planteó como una extensión del trabajo que desde

2 Programa Individualizado de Cuidado y Valoración del Desarrollo, 
1999 se estaba haciendo, en conjunto con kinesiología, en el seguimiento y estimulación de niños prematuros nacidos en el Hospital Guillermo Grant Benavente de Concepción (12). De la evaluación de este trabajo había surgido la necesidad de ir más allá de lo rehabilitador, ampliando la labor de terapia ocupacional hacia el área preventiva y con este fin pareció natural la estrategia de proyectarse hacia un trabajo con las madres mientras los bebés permanecían aún hospitalizados en la Unidad de Neonatología.

En sus comienzos la intervención de terapia ocupacional se centró en aquellas acciones que decían relación directa con las madres: Previo al alta entregar información acerca de la necesidad de proveer un cuidado especial a su hijo por su condición de prematuridad, dar directrices en relación a cómo implementar este cuidado y motivar para su posterior incorporación al programa de seguimiento en modalidad ambulatoria.

En orden a favorecer un mejor desarrollo psicomotor de los niños en el mediano y largo plazo, desde fines de 2005 la intervención se amplió hacia un trabajo directo con los bebés hospitalizados, incorporando las siguientes acciones efectuadas por terapeuta ocupacional:

- Evaluación de características neuroconductuales del niño, en base a observación del mismo en momentos de descanso y actividad (por ejemplo durante la alimentación).

- Colaboración en medidas de control de estímulos ambientales, las cuales son parcialmente implementadas por personal de enfermería.

- Cuidado de posiciones, como un complemento a las acciones de personal de enfermería en esta línea.

- Indicaciones a la madre para adecuar su acercamiento al niño de acuerdo a edad gestacional, condición médica y características del mismo.

- Indicaciones a la madre al momento del alta.

Estas acciones, además de su efecto benéfico directo para los bebés, en los cuales se observa en control posterior de desarrollo psicomotor un discreto aumento en sus puntajes de normalidad así como menor incidencia de signos neurológicos blandos, ejercen también un efecto positivo en las madres, para las cuales resulta una ayuda toda acción dirigida a hacerlas sentir más seguras respecto del cuidado de este frágil y especial hijo.

En los últimos 2 años se han incorporado, a las acciones ya mencionadas, las siguientes:

- Estimulación táctil en forma de tacto profundo.

- Estimulación propioceptiva: contención y facilitación de movimientos organizados por parte del niño.

- Estimulación vestibular.

- Indicaciones a la madre para aplicar dicha estimulación.

\section{MÉTOdo}

La intervención de terapia ocupacional se estructura de acuerdo a la siguiente metodología:

a) Intervención de acuerdo a edad gestacional: tanto por consideración a las características neurológicas de los bebés prematuros, como por la organización funcional de la unidad de neonatología, las acciones se inician no antes de las 30 semanas de edad gestacional.

b) Cuidado de la condición de salud del niño: si la condición de salud del niño es inestable o se encuentra francamente deteriorada, sólo se efectúan acciones orientadas al cuidado de posiciones y a instruir y apoyar a la madre.

c) Respetar el descanso del bebé: si en el momento en que se presenta la terapeuta el bebé se encuentra en estado de sueño profundo, se le deja dormir y no se efectúan intervenciones directas.

d) Tener en mente los principios teóricos generales que explican las limitaciones y capacidades de autorregulación del bebé. 
e) Atención permanente a las señales del bebé, teniendo la capacidad de interpretarlas como recepción o rechazo del estímulo.

La intervención se inicia desde que el niño cumple las 30 semanas de gestación y en la medida que su condición médica lo permita. En cuanto es posible se efectúan en forma diaria todas las acciones que suponen una intervención directa de la terapeuta hacia el niño. El trabajo con las madres se organiza en etapas, entregando información e indicaciones específicas en forma individualizada y gradual, de acuerdo a la edad gestacional del niño. Luego de su alta los niños asisten a controles mensuales de terapia ocupacional en forma ambulatoria.

\section{Resultados}

Durante la etapa ambulatoria se continúa la atención de esta población de niños con la aplicación de acciones orientadas a evaluar su desarrollo psicomotor, aplicar técnicas de estimulación neurosensorial global, aplicar tratamiento rehabilitador cuando la condición del niño lo requiera y entregar consejería a la familia en relación a cuidado del niño y normas de crianza.

El nivel de desarrollo psicomotor se evalúa de acuerdo a la edad corregida del niño y para ello se utiliza la Escala de Evaluación del Desarrollo Psicomotor de Soledad Rodríguez (EEDP). Esto se complementa con observación de manifestaciones de la capacidad de modulación sensorial del niño, así como de la posible presencia de signos neurológicos blandos.

A continuación se presentan los resultados de evaluación de desarrollo psicomotor de los niños nacidos entre 2006 y primeros meses de 2007, período en el cual se comenzó a estructurar la intervención directa de terapia ocupacional en la unidad de neonatología.
Tabla N 1: Desarrollo PM a los 6 meses de edad corregida, en niños de 29 semanas de gestación y menos

\begin{tabular}{|c|c|c|}
\cline { 2 - 3 } \multicolumn{1}{c|}{} & $\mathbf{N}$ & $\%$ \\
\hline Normal & 16 & $43 \%$ \\
\hline Riesgo & 16 & $43 \%$ \\
\hline Retraso & 5 & $14 \%$ \\
\hline TOTAL & 37 & $100 \%$ \\
\hline
\end{tabular}

Tabla N²: Desarrollo PM a los 12 meses de edad corregida, en niños de 29 semanas de gestación y menos

\begin{tabular}{|c|c|c|}
\cline { 2 - 3 } \multicolumn{1}{c|}{} & $\mathbf{N}$ & $\%$ \\
\hline Normal & 18 & $49 \%$ \\
\hline Riesgo & 16 & $43 \%$ \\
\hline Retraso & 3 & $8 \%$ \\
\hline TOTAL & 37 & $100 \%$ \\
\hline
\end{tabular}

Las tablas $\mathrm{N}^{\mathrm{a}} 1$ y $\mathrm{N}^{\mathrm{a}} 2$ muestran los resultados obtenidos en su evaluación de desarrollo psicomotor a los 6 y a los 12 meses de edad corregida por un grupo de 37 niños nacidos con 29 semanas de gestación o menos, los que fueron atendidos en la unidad de neonatología y al momento de su alta continuaron sus controles en el Servicio de Medicina Física y Rehabilitación. El número de niños atendidos en neonatología es mayor, pero por múltiples razones no todos ellos continúan sus controles en la etapa ambulatoria, o asisten en forma esporádica.

Estos resultados permiten observar que en este grupo de niños hay una mejoría en su nivel de desarrollo psicomotor al progresar en edad, con un aumento del porcentaje de niños con resultado Normal y una disminución del porcentaje de niños con resultado de Retraso a los 12 meses de edad corregida. 
Tabla N 3: Desarrollo PM a los 6 meses de edad corregida en niños de 30 a 32 semanas de gestación

\begin{tabular}{|c|c|c|}
\cline { 2 - 3 } \multicolumn{1}{c|}{} & $\mathbf{N}$ & $\%$ \\
\hline Normal & 18 & $46 \%$ \\
\hline Riesgo & 17 & $44 \%$ \\
\hline Retraso & 4 & $10 \%$ \\
\hline TOTAL & 39 & $100 \%$ \\
\hline
\end{tabular}

Tabla N ${ }^{\circ}$ 4: Desarrollo PM a los 12 meses de edad corregida en niños de 30 a 32 semanas de gestación

\begin{tabular}{|c|c|c|}
\cline { 2 - 3 } \multicolumn{1}{c|}{} & $\mathbf{N}$ & $\%$ \\
\hline Normal & 24 & $62 \%$ \\
\hline Riesgo & 12 & $31 \%$ \\
\hline Retraso & 3 & $8 \%$ \\
\hline TOTAL & 39 & $100 \%$ \\
\hline
\end{tabular}

Las tablas $\mathrm{N}^{\circ} 3$ y $\mathrm{N}^{\circ} 4$ muestran los resultados obtenidos en su evaluación del desarrollo psicomotor por un grupo de 39 niños nacidos con edades gestacionales entre 30 y 32 semanas. También en este grupo se observa una evidente mejoría en su nivel de desarrollo psicomotor al progresar en edad, siendo posible acá apreciar porcentajes más altos de resultado Normal a los 6 y a los 12 meses de edad corregida comparado con el grupo de niños nacidos de 29 semanas gestacionales y menos.

Junto con lo anterior, en observación global es posible constatar, en la mayoría de los niños a lo largo del primer año de edad corregida, una disminución de signos neurológicos blandos (retracción escapular, hipertono, tendencia a mayor uso de mano derecha, etc). Esto, en términos generales, se produce con independencia de la edad gestacional al nacer.

A continuación, en las tablas $N^{0} 5$ y $N^{0} 6$ se exponen los resultados obtenidos a los 6 meses de edad corregida en un grupo de niños nacidos entre los años
2008-2009. Tal como se mencionó con anterioridad, a partir de este período se enriqueció la intervención de terapia ocupacional, complementándola con acciones de estimulación específicas.

Tabla No 5: Desarrollo PM a los 6 meses de edad corregida en niños nacidos de 29 semanas de gestación y menos

\begin{tabular}{|c|c|c|}
\cline { 2 - 3 } \multicolumn{1}{c|}{} & $\mathbf{N}$ & $\%$ \\
\hline Normal & 7 & $53,8 \%$ \\
\hline Riesgo & 5 & $38,5 \%$ \\
\hline Retraso & 1 & $7,7 \%$ \\
\hline TOTAL & 13 & $100 \%$ \\
\hline
\end{tabular}

Tabla No 6: Desarrollo PM a los 6 meses de edad corregida en niños nacidos entre 30 y 32 semanas de gestación

\begin{tabular}{|c|c|c|}
\cline { 2 - 3 } \multicolumn{1}{c|}{} & $\mathbf{N}$ & $\%$ \\
\hline Normal & 8 & $58 \%$ \\
\hline Riesgo & 3 & $21 \%$ \\
\hline Retraso & 3 & $21 \%$ \\
\hline TOTAL & 14 & $100 \%$ \\
\hline
\end{tabular}

Es posible apreciar acá que a la edad de 6 meses de edad corregida ambos grupos, tanto aquellos niños nacidos de 29 semanas gestacionales y menos como los nacidos entre 30 y 32 semanas, exhiben mejores niveles de desarrollo psicomotor, con mayores porcentajes de resultados en nivel Normal, comparados con los grupos estudiados previamente. Resulta Ilamativo además observar que en el grupo de niños de menor edad gestacional se aprecia menor porcentaje que presenta una condición de retraso del desarrollo psicomotor. También en estos grupos ha sido posible constatar, además de su buen nivel de desarrollo, disminución gradual de signos neurológicos blandos. 


\section{Ejemplo de caso clínico}

- R.F.P.

- $\quad$ Fecha de nacimiento: 15/10/09.

- $\quad$ Edad gestacional: 26 semanas.

- Peso al nacer: 680 grs.

- $\quad$ APGAR : 1-1-2-2, 7 a los 13 minutos de nacido.

- Diagnósticos: enfermedad de membrana hialina, retinopatía del prematuro; pequeño para la edad gestacional; enterocolitis necrotizante.

- Evoluciona con una condición médica inestable que obliga a continuos traslados a sala de cuidados intensivos, permaneciendo hospitalizado durante aproximadamente 5 meses luego de su nacimiento.

- Se inició la intervención de terapia ocupacional luego de su traslado a sala de cuidados medios, contando con 36 semanas de edad gestacional.

- Se continúan sus controles en forma ambulatoria luego del alta, la que se concreta a los 2 meses de edad corregida.

- Desarrollo psicomotor a los 2 meses 10 días de edad corregida: 0.64 - retraso.

- Desarrollo psicomotor a los 5 meses de edad corregida: 0.83 - riesgo.

- $\quad$ Resultado ampliamente satisfactorio a la luz de los antecedentes del menor, así como por tratarse de un niño de procedencia rural.

- Actualmente continúa en controles regulares en nuestro servicio.

\section{ANÁLISIS DE RESULTADOS}

nuestro trabajo en neonatología se desarrolla en un contexto que supone algunas limitantes para su aplicación metódica y ulterior evaluación de resultados, a saber:

- Dada la estructuración administrativa del Servicio de Medicina Física y Rehabilitación, al que pertenece la profesional, así como la necesidad de responder a requerimientos de agenda y compro- misos de gestión, el horario hasta ahora dispuesto para la colaboración en neonatología es parcial.

- Por el hecho de ser el Hospital Guillermo Grant Benavente un centro de referencia de la zona sur, así como por las características demográficas de la región, numerosos padres se ven imposibilitados de acudir en forma diaria a visitar a sus hijos hospitalizados, limitándose su posibilidad de contacto con esta profesional. Algunos bebés derivados de otras regiones, son visitados en forma esporádica, o en ocasiones, nunca.

- La Escala de Evaluación del Desarrollo Psicomotor de Soledad Rodríguez, siendo el instrumento oficial utilizado en nuestro país para evaluar los primeros años de vida, no evalúa la totalidad de aspectos del desarrollo neurológico y motor. Ha surgido desde tiempo atrás la necesidad de contar con otros instrumentos que puedan dar cuenta en forma más acabada de las diferentes expresiones del desarrollo. Tal es el caso del Test de Bayley, el cual, a pesar de no estar estandarizado para nuestra población infantil, constituye hoy en día la "regla de oro" para evaluar el desarrollo en los tres primeros años de vida. Sin embargo, no se dispone de este instrumento en nuestro servicio.

- Al evaluar el desarrollo de los niños prematuros controlados en Medicina Física y Rehabilitación es muy difícil determinar si sus logros se derivan exclusivamente de las intervenciones descritas, o se relacionan también, como posiblemente sucede, con otras acciones implementadas tanto durante la hospitalización (técnica de madre canguro aplicada por matrona, u otros) como con posterioridad al alta (manejo kinésico en el Servicio de Medicina Física, acciones derivadas del programa Chile Crece Contigo, etc.). Por lo tanto no es posible afirmar en forma categórica que los resultados mostrados se relacionan directamente con la intervención de terapia ocupacional.

Tenemos, sin embargo, la certeza de que el programa descrito se traduce en beneficios para estos niños. 
Las complejidades analizadas son un estímulo para optimizar y sistematizar en mayor medida su aplicación, procurando definir medidas que nos permitan objetivar los progresos mostrados por los bebés, tanto en la etapa intra hospitalaria (por ejemplo estabilidad fisiológica, aumento de peso, disminución de estadía hospitalaria) como extra hospital (desarrollo psicomotor, disminución de signos neurológicos blandos, optimización del desempeño ocupacional en edad preescolar y escolar).

\section{CONCLUSIONES}

En Chile el 1\% de los nacimientos son prematuros de menos de 1500 grs., lo que corresponde a 2500 niños, de los cuales sobreviven 2000. Estos antecedentes son un Ilamado a la acción, más aún teniendo presente que existe preocupación a nivel de las autoridades de salud por lo insuficiente de los programas de habilitación y rehabilitación destinados a esta población (2).

Para los terapeutas ocupacionales es indispensable iniciar un diálogo en base al intercambio de experiencias relativas a la atención de bebés prematuros, con el fin de diseñar programas de intervención más efectivos, a la vez que se facilita la especialización y preparación que el trabajo en estos centros requiere. Es necesario que nos planteemos una constante puesta en común, análisis y reflexión acerca de nuestro trabajo en esta área en particular, tanto con el propósito de contribuir al logro de los mejores niveles de desarrollo integral de estos niños, como de potenciar nuestro progreso y posicionamiento profesional.

Así mismo, debemos incorporarnos a los equipos profesionales de las Unidades de Neonatología, lo que se traducirá en un enriquecimiento mutuo en beneficio de nuestros usuarios. La presencia de terapia ocupacional a este nivel está planteada por la propia autoridad sanitaria de nuestro país. En los estándares para la Organización de la Red Chilena de Neonatología (13) se incluye a estos profesionales como integrantes del equipo con que deberían contar los centros de alta complejidad para atención de neonatos.
La interacción y coordinación con otros especialistas y programas tanto a nivel hospitalario como de la comunidad deberían constituirse en una meta a lograr, con el fin de potenciar todos los esfuerzos efectuados en beneficio de nuestros niños. En este sentido, concordamos con la propuesta de adaptar programas a nuestra realidad local más que limitar nuestro quehacer por la eventual falta de recursos (14).

La familia debe ser considerada dentro del equipo que atiende al bebé y con este fin ser incorporada desde los primeros días de vida del niño. Existe suficiente evidencia de que un enfoque centrado en la familia contribuirá a minimizar los efectos negativos de la hospitalización, a la vez que proveerá a los padres la percepción de competencia en el manejo de su hijo. Facilitar su presencia como parte del equipo tiene además la ventaja de contribuir a desarrollo vincular entre el bebé y sus padres (15), tarea que no es ajena al quehacer del terapeuta ocupacional, en cuanto nos compete contribuir al fortalecimiento del sistema familiar como condición para el desarrollo integral de los bebés.

\section{ReferenCias BibliográficAs}

(1) HUBNER, M.E. "Sobrevida, viabilidad y pronóstico del prematuro". Revista Médica de Chile, 2002; 130: 931-938.

(2) MORGUES, M. Informe Técnico: Recién Nacidos con menos de 32 semanas de edad gestacional. SNSS de Chile, Quinquenio 2000-2004, MINSAL.

(3) CONNORS, M. "Motor Outcomes in Premature Infants", NBIN 2003; 3: 104-109.

(4) FOULDER-HUGHES, L. "Do Mainstream schoolchildren who were born preterm have motor problems?". British Journal of Occupational Therapy, 2003; 66: 9-16.

(5) BRUGGINK, J. "The early motor repertoire of children born preterm is associated with intelligence al school age". Pediatrics, Mayo 2010. 
(6) NARBERHAUS, A. : Trastornos neuropsicológicos y del neurodesarrollo en el prematuro, Anales de Psicología. Publicación de la Universidad de Murcia. 2004; 20: 317-326.

(7) GIBBS, D. y BOSHOFF, K.: Understanding parenting occupations in neonatal intensive care: application of the Person-Environment-Occupation Model. British Journal of Occupational Therapy, Febrero 2010; 9: 55-63.

(8) HUNTER, J. Neonatal Intensive Care Unit. En Jane Case-Smith: "Occupational Therapy for Children", 2005, pág. 689-769.

(9) Apuntes del curso "Estrategias basadas en la evidencia para la promoción del desarrollo cerebral en el recién nacido pre-término", organizado por la Escuela de Post Grado de la Facultad de Medicina de la Universidad de Chile, 2007.

(10) ALS, H. y col. Early experience alters brain function and structure. Pediatrics, 113: 846-857. 2004.
(11) GORSKi, P., BRAZELTON, T. Stages of behavioural organization in the high-risk neonate: theoretical clinical considerations. Seminars in Perinatologý, 1979; 3: 61-72.

(12) AVILES, C., MADARIAGA, P. y cols. Intervención temprana en prematuros: una experiencia de trabajo. En Revista Chilena de Terapia Ocupacional, $N^{\mathrm{a}}$ 4, 2004.

(13) MINSAL: "Standares y Organización de la Red Neonatal Chilena", Programa de Seguimiento del Prematuro, 2010.

(14) MIRA, A. Terapia ocupacional neonatal, una propuesta para la acción. Revista Chilena de Terapia Ocupacional, Nª 6, 2006.

(15) MIRA, A. La importancia de las relaciones tempranas y el apego en los niños con necesidades especiales. Revista Chilena de Terapia Ocupacional, Nª 9, 2009. 